\title{
Elastic properties of polymer interfaces: Aggregation of pure diblock, mixed diblock, and triblock copolymers
}

\author{
M. Müller ${ }^{1,2}$ and G. Gompper ${ }^{2}$ \\ ${ }^{1}$ Institut für Physik, WA 331, Johannes Gutenberg Universität, D-55099 Mainz, Germany \\ ${ }^{2}$ Forschungszentrum Jülich, Institut für Festkörperforschung, D-52425 Jülich, Germany
}

(Received 30 May 2002; published 21 October 2002)

\begin{abstract}
Block copolymers adsorbing to an interface between two immiscible homopolymers modify the elastic constants of this interface. Within self-consistent field calculations for Gaussian chains, we determine how the bending constants vary in dependence on the block copolymer concentration and architecture. Four phenomena are discussed. (i) When a tricritical or isotropic Lifshitz critical point is approached in a ternary mixture by varying the concentration of diblock copolymers or changing temperature, the elastic constants vanish. We determine the corresponding power laws, and show that the de Gennes-Taupin criterium for the stability of lamellar phases against undulations and the Ginzburg-Landau criterium for bulk fluctuations yield identical predictions for the validity of the mean-field approximation. (ii) Addition of a small amount of diblock copolymers modifies the bending rigidity. If the diblock copolymers are comparable in length to the homopolymers, adsorption of the diblocks reduces the bending rigidity. If the diblocks are much longer, they increase the bending rigidity. Only for an extreme ratio of chain lengths $(>100)$, the predictions for polymers tethered to an infinitely thin, impenetrable sheet become accurate. (iii) Mixtures of short and long symmetric diblock copolymers are studied, as well as mixtures of two asymmetric diblocks, which are obtained by exchanging the long and short ends. The saddle-splay modulus is found to be the same in both mixtures, while the bending rigidity is significantly smaller in the latter case. (iv) The role of the block copolymer architecture is studied by comparing the effect of triblock copolymers with the effect of diblocks with the same overall length and composition. We propose that triblock copolymers are a very efficient way to control the spontaneous curvature of an interface.
\end{abstract}

DOI: 10.1103/PhysRevE.66.041805

PACS number(s): 61.25.Hq, 68.05.-n, 62.20.Dc

\section{INTRODUCTION}

Many qualitative features of homopolymer-copolymer blends are shared by mixtures of oil, water, and surfactants, or by mixtures of biologically relevant lipids and water. For instance, all these systems exhibit a similar topology of the phase diagram, which is brought about by the "amphiphilic" character of the copolymer, the surfactant, or the lipid. In both of the first two cases, it is technologically highly desirable to obtain macroscopically uniform mixtures of all three components with an amount of copolymer or surfactant as small as possible. Furthermore, the behavior of these systems is controlled by the same physical mechanisms, which are the low interfacial tension and the curvature elasticity of the decorated interfaces between the two components to be solubilized, as well as the direct interactions between these interfaces.

Adding diblock copolymers to a blend of incompatible homopolymers improves the application properties of polymeric "alloys." Therefore the phase behavior, and interface structure has attracted abiding interest. Self-consistent field calculations [1-3] predict a rich phase behavior including highly swollen lamellar phases and a three phase coexistence between uniform homopolymer-rich phases and a lamellar copolymer-rich phase. Polymeric surfactants offer particular advantages as model systems to investigate this universal behavior, because (i) the interface structure itself can be described by a few mesoscopic parameters (e.g., incompatibility per molecule $\chi N$ and chain extension $R_{e}$ ) and (ii) fluc- tuation effects are reduced due to the large extension of the molecules.

Two recent discoveries shed more light onto the close relation between amphiphilic systems and polymer mixtures. The first is the discovery of polymeric microemulsions, i.e., of an isotropic phase in polymer mixtures, in which the homopolymers form a disordered array of intertwined, microphase-separated channel networks [4-9]. This phase has the same structure as the bicontinuous microemulsions in amphiphilic systems, which have been investigated intensively over many years. The second is the addition of an amphiphilic block copolymer to a oil-water microemulsion was found to have drastic consequences on its swelling behavior $[10,11]$. Already a small amount of block copolymer was found to increase the efficiency of the surfactant enormously. This effect can be understood as a result of the changed curvature elasticity of the decorated oil-water interface.

It therefore seems possible to also control the phase behavior of polymer blends by adding a second type of block copolymer, or by changing the copolymer architecture. We want to investigate in this paper several possibilities to modify the curvature elasticity of the polymer interface by block copolymers and block-copolymer mixtures. A special case, the bidisperse mixture of symmetric copolymers with equal interfacial excesses of short and long chains, has been investigated in Ref. [12], and has been found advantageous for stabilizing polymeric microemulsions.

On a mesoscopic length scale the properties of interfaces between coexisting phases or self-assembled aggregates are often described by an effective interface Hamiltonian. Such 
models have proven useful in the context of wetting phenomena [13], biological membranes, and microemulsions [1416]. In the framework of an effective interface Hamiltonian, the interface is conceived as an infinitely thin sheet, which is characterized by a few effective parameters: the interface tension $\sigma$, the spontaneous curvature $\lambda$, the bending rigidity $\kappa$, and the saddle-splay modulus $\bar{\kappa}$. The Hamiltonian of such a sheet takes the form [17]

$$
\mathcal{H}=\int d S\left\{\sigma+\lambda H+2 \kappa H^{2}+\bar{\kappa} G\right\}
$$

where $H=\left(c_{1}+c_{2}\right) / 2$ and $G=c_{1} c_{2}$ are the local mean and Gaussian curvatures. In spite of its apparent simplicity, the statistical mechanics of such an interface is quite intricate and the model exhibits a rich phase behavior [14-16].

The parameters $\sigma, \lambda, \kappa$, and $\bar{\kappa}$ are input into the effective interface description. They have to be calculated from a microscopic model, which incorporates more details of the interface. In the following we calculate the elastic properties of interfaces in a mixture of homopolymers and diblock copolymers. The elastic properties of polymer interfaces have been calculated in the framework of the self-consistent field theory by Matsen $[18,19,12]$ and Laradji $[20]$. In the following, we use the computational method of Matsen [18] to calculate the effective parameters of the effective interface Hamiltonian in the framework of a well-established coarsegrained model.

After a brief outline of the model and the method, we focus on four topics.

(i) We study the behavior of the bending rigidity and the saddle-splay modulus upon approaching an isotropic Lifshitz or tricritical Lifshitz point. Mean field theory fails "dramatically" [9] in the vicinity of those points, and fluctuations give rise to a disordered microemulsionlike structure.

(ii) We investigate how a small amount of copolymer modifies the elastic constants of the interface and compare our results to analytical predictions [21-23] of long chains attached to an impenetrable infinitely thin interface.

(iii) Comparing mixtures of symmetric and asymmetric diblock copolymers, we verify explicitly how exchanging asymmetric amphiphiles from one side of the curved interface to the other (flip-flop) affects the bending rigidity and we relate our results to membrane models [24-27].

(iv) The role of architecture is illustrated by investigating symmetric $A B A$-triblock copolymers with diblock copolymers of identical composition. It is shown that the middle block does not impart a spontaneous curvature onto the interface.

\section{POLYMER MODEL AND SELF-CONSISTENT FIELD CALCULATIONS}

The technical details of our calculations are very similar to previous work by Matsen [18]. The technique has proven to be more accurate than alternative approaches. We give here only a brief account which also serves to introduce our notation. We consider a quaternary mixture of two homopolymers and two diblock copolymers, which consist of
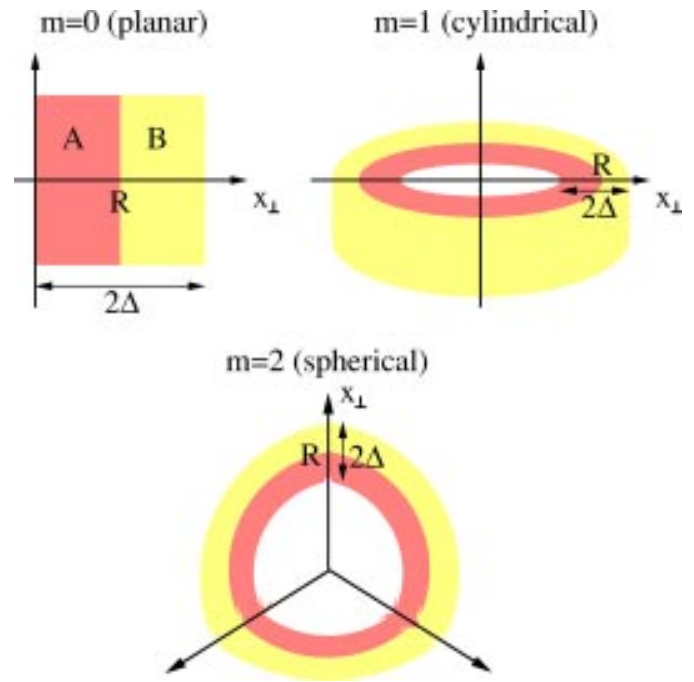

FIG. 1. Illustration of the planar $(m=0)$, cylindrical $(m=1)$, and spherical $(m=2)$ geometry used in the self-consistent field (SCF) calculations.

monomer species $A$ and $B$. Both homopolymers have equal chain length $N$, and $N / \alpha_{1}$ and $N / \alpha_{2}$ denote the chain lengths of the two copolymers. The first diblock copolymer contains $f_{1} N / \alpha_{1} A$ segments and the second one comprises $f_{2} N / \alpha_{2} A$ segments. The Flory-Huggins parameter $\chi$ describes the repulsion of the different monomer species. All chains are modeled as Gaussian chains with identical statistical segment length, the end-to-end distance of the homopolymers and the two diblock copolymers are $R_{e}$, $R_{e} / \sqrt{\alpha_{1}}$, and $R_{e} / \sqrt{\alpha_{2}}$, respectively.

We calculate the free energy of an $A B$ interface in three geometries-planar $(m=0)$, cylindrical $(m=1)$, and spherical $(m=2)$ as illustrated in Fig. 1. Let $x_{\perp}$ denote the coordinate normal to the interface. The interface is centered at $x_{\perp}=R$ and we calculate the properties inside the shell $R$ $-\Delta \leqslant x_{\perp} \leqslant R+\Delta$. The width $\Delta$ is chosen large enough for the profiles to reach their bulk values at the boundaries. Our calculations are performed in the grand-canonical ensemble, but we specify explicitly the location of the interface [28]. The grand-canonical free energy of the mixture of two homopolymers and two diblock copolymers takes the form

$$
\begin{aligned}
\frac{N \mathcal{G}}{k_{B} T \rho}= & -z_{h A} \mathcal{V} Q_{h A}-z_{h B} \mathcal{V} Q_{h B}-z_{1} \mathcal{V} Q_{1}-z_{2} \mathcal{V} Q_{2} \\
& +\int d \mathbf{r} \chi N \phi_{A}(\mathbf{r}) \phi_{B}(\mathbf{r})-\int d \mathbf{r}\left\{w_{A}(\mathbf{r}) \phi_{A}(\mathbf{r})\right. \\
& +w_{B}(\mathbf{r}) \phi_{B}(\mathbf{r})+\xi(\mathbf{r})\left[1-\phi_{A}(\mathbf{r})-\phi_{B}(\mathbf{r})\right] \\
& \left.-\psi \Delta \delta\left(x_{\perp}-R\right)\left[\phi_{A}(\mathbf{r})-\phi_{B}(\mathbf{r})\right]\right\} .
\end{aligned}
$$

$\phi_{A}$ and $\phi_{B}$ denote the local composition of the mixture and $\rho$ the monomer number density. $\mathcal{V}$ denotes the volume of the system. $T$ is the temperature and $k_{B}$ the Boltzmann constant. $z_{A}, z_{B}, z_{1}$, and $z_{2}$ are the fugacities of the polymers. They are related to the excess chemical potentials via $z_{A}$ $=\exp \left(\mu_{A}^{\mathrm{exc}} / k_{B} T\right)$, and similar relations hold for the other 
polymers. Since the system is incompressible, $\phi_{A}(\mathbf{r})$ $+\phi_{B}(\mathbf{r})=1$, there are only three independent chemical potentials and we set $z_{A}=1 . \xi(\mathbf{r})$ and $\psi$ are Lagrange multipliers to enforce incompressibility and to pin the center of the interface, where $\phi_{A}=\phi_{B}$, at the surface $x_{\perp}=R . \mathcal{Q}_{A}$, $\mathcal{Q}_{B}, \mathcal{Q}_{1}$, and $\mathcal{Q}_{2}$ are the single chain partition functions [29] of the two homopolymers $A$ and $B$ and the two copolymers in the external fields, respectively.

$$
\begin{aligned}
\mathcal{Q}_{1}\left[w_{A}, w_{B}\right]= & \frac{1}{\mathcal{V}} \int \mathcal{D}[\{\mathbf{r}(s)\}] \exp \left(-\frac{3}{2 R_{e}^{2}} \int_{0}^{1 / \alpha_{1}} d s\left[\frac{d \mathbf{r}(s)}{d s}\right]^{2}\right. \\
& \left.-\int_{0}^{f_{1} / \alpha_{1}} d s w_{A}(\mathbf{r}(s))-\int_{f_{1} / \alpha_{1}}^{1 / \alpha_{1}} d s w_{B}(\mathbf{r}(s))\right)
\end{aligned}
$$

and similarly for the other polymers. $w_{A}$ is the external field which acts on the $A$ segments and $w_{B}$ is the external field which acts on the $B$ segments. The mean-field approximation consists in replacing the densities and external fields by their most probable values. These are given by the self-consistent set of equations,

$$
\begin{gathered}
w_{A}-w_{B}=-\chi N\left(\phi_{A}-\phi_{B}\right)-2 \psi \Delta \delta\left(x_{\perp}-R\right), \\
\phi_{A}+\phi_{B}=1, \\
\phi_{A}\left(x_{\perp}=R\right)=\phi_{B}\left(x_{\perp}=R\right), \\
\phi_{A}=z_{h A} \mathcal{V} \frac{\mathcal{D} Q_{h A}}{\mathcal{D} w_{A}}+z_{1} \mathcal{V} \frac{\mathcal{D} Q_{1}}{\mathcal{D} w_{A}}+z_{2} \mathcal{V} \frac{\mathcal{D} Q_{2}}{\mathcal{D} w_{A}}, \\
\phi_{B}=z_{h B} \mathcal{V} \frac{\mathcal{D} Q_{h B}}{\mathcal{D} w_{B}}+z_{1} \mathcal{V} \frac{\mathcal{D} Q_{1}}{\mathcal{D} w_{B}}+z_{2} \mathcal{V} \frac{\mathcal{D} Q_{2}}{\mathcal{D} w_{B}} .
\end{gathered}
$$

In the grand-canonical ensemble $\mathcal{Q}_{A}=\bar{\phi}_{h A} / z_{A}$, where $\bar{\phi}_{h A}$ is the average composition of $A$ homopolymers. Similar relations hold for $B$ homopolymers and copolymers.

To calculate the segment densities it is useful to introduce end segment distributions [29]. They obey modified diffusion equations, which take a particularly convenient form in Fourier representation. Following Matsen [18], we expand all spatial dependencies normal to the interface in a cos series with $M$ terms. For example, the $A$ monomer density takes the form $\phi_{A}\left(x_{\perp}\right)=\sum_{i=1}^{M} \phi_{A i} f_{i}\left(x_{\perp}\right)$ with

$$
\begin{gathered}
f_{i}\left(x_{\perp}\right)=\mathcal{N}_{i} \cos \left(\frac{(i-1) \pi\left(x_{\perp}-R+\Delta\right)}{2 \Delta}\right) \\
\mathcal{N}_{1}=1 \quad \text { and } \quad \mathcal{N}_{i}=\sqrt{2} \quad \text { otherwise. }
\end{gathered}
$$

Up to $M=360$ basis functions have been used in our calculations.

The bending moduli are extracted from the dependence of the excess interface free energy on the curvature. Using the dimensionless curvature $C=R_{e} / R$, we obtain [18]

$$
\begin{aligned}
g & \equiv \frac{N\left(\mathcal{G}-\mathcal{G}_{\text {bulk }}\right)}{k_{B} T \rho \mathcal{A} R_{e}}=\frac{\Delta \mathcal{G}}{\sqrt{\bar{N}} k_{B} T\left(\mathcal{A} / R_{e}^{2}\right)} \\
& = \begin{cases}\Sigma+\frac{1}{2} \Lambda C+\frac{1}{2} K C^{2} & \text { for } m=1 \\
\Sigma+\Lambda C+(2 K+\bar{K}) C^{2} & \text { for } m=2,\end{cases}
\end{aligned}
$$

where $\mathcal{A}$ denotes the interface area and $\mathcal{G}_{\text {bulk }}$ is the grandcanonical free energy of the corresponding homogeneous system. Comparing expression (10) with Eq. (1) we identify

$$
\begin{array}{cc}
\Sigma=\sigma R_{e}^{2} / k_{B} T \sqrt{\bar{N}}, \quad \Lambda=\lambda R_{e} / k_{B} T \sqrt{\bar{N}}, \\
K=\kappa / k_{B} T \sqrt{\bar{N}}, \quad \bar{K}=\bar{\kappa} / k_{B} T \sqrt{\bar{N}} .
\end{array}
$$

The quantity $\bar{N}=\left(\rho R_{e}^{3} / N\right)^{2}$ measures the degree of interdigitation of the polymers and it scales like the chain length.

The properties of the homogeneous system are given by

$$
\begin{gathered}
\frac{\bar{\phi}_{1} \alpha_{1}}{z_{1}}=\left(\frac{\bar{\phi}_{h A}}{z_{A}}\right)^{f_{1} / \alpha_{1}}\left(\frac{\bar{\phi}_{h B}}{z_{B}}\right)^{\left(1-f_{1}\right) / \alpha_{1}}, \\
\frac{\bar{\phi}_{2} \alpha_{2}}{z_{2}}=\left(\frac{\bar{\phi}_{h A}}{z_{A}}\right)^{f_{2} / \alpha_{2}}\left(\frac{\bar{\phi}_{h B}}{z_{B}}\right)^{\left(1-f_{2}\right) / \alpha_{2}}, \\
\bar{\phi}_{A}=\bar{\phi}_{h A}+f_{1} \bar{\phi}_{1}+f_{2} \bar{\phi}_{2}, \\
\bar{\phi}_{B}=\bar{\phi}_{h B}+\left(1-f_{1}\right) \bar{\phi}_{1}+\left(1-f_{2}\right) \bar{\phi}_{2}, \\
\chi N\left(\bar{\phi}_{A}-\bar{\phi}_{B}\right)=\ln \frac{\bar{\phi}_{h A} z_{B}}{z_{A} \bar{\phi}_{h B}},
\end{gathered}
$$

and

$$
\begin{aligned}
\frac{N \mathcal{G}_{\text {hom }}}{k_{B} T \rho \mathcal{V}}= & -\bar{\phi}_{h A}-\bar{\phi}_{h B}-\bar{\phi}_{1} \alpha_{1}-\bar{\phi}_{2} \alpha_{2}+\chi N \bar{\phi}_{A} \bar{\phi}_{B} \\
& -\bar{\phi}_{A} \ln \frac{\bar{\phi}_{h A}}{z_{A}}-\bar{\phi}_{B} \ln \frac{\bar{\phi}_{h B}}{z_{B}},
\end{aligned}
$$

where $\bar{\phi}_{h A}, \bar{\phi}_{h B}, \bar{\phi}_{1}$, and $\bar{\phi}_{2}$ denote the composition of the homopolymers and copolymers in the bulk. The monomer excess of copolymer 1 at an interface is calculated via [18]

$$
\begin{aligned}
\Omega_{1}= & \frac{1}{\mathcal{A} R_{e}} \int d \mathbf{r} \phi_{1}(\mathbf{r})-\frac{\bar{\phi}_{1}^{(1)} R}{m+1}\left\{1-\left(1-\frac{\Delta}{R}\right)^{m+1}\right\} \\
& -\frac{\bar{\phi}_{1}^{(2)} R}{m+1}\left\{\left(1+\frac{\Delta}{R}\right)^{m+1}-1\right\},
\end{aligned}
$$

where $\bar{\phi}_{1}^{(1)}$ and $\bar{\phi}_{1}^{(2)}$ denote the composition of copolymer 1 in the two coexisting bulk phases. The excess number of 
copolymer chains per unit area of the interface is $\Omega_{1} \sqrt{\bar{N}} \alpha_{1} / R_{e}^{2}$. Again, a similar expression holds for the second copolymer.

The copolymers segregate to the interface as to extend their blocks into the appropriate homopolymer-rich phase. Segregation reduces the interface free energy. For $\Omega_{1} \ll 1$ the homopolymers interdigitate the copolymer brush at the interface (wet brush), and the copolymers basically do not interact with each other. As we increase the number of copolymers, the copolymer density at the interface increases and the homopolymers are expelled from the center of the interface (dry brush, $\Omega \gg 1$ ). The copolymers stretch perpendicular to the interface as to comply with the incompressibility constraint. Of course, there is no sharp transition between the wet and dry brush regime, but a rather gradual crossover. As we increase the fugacity of copolymers further, eventually, the copolymers will segregate from the homopolymers and will form a (lamellar) microphase. This possibility is not considered further here, but we restrict our attention to the segregation of copolymers to the interface between phases, which are rich in $A$ or $B$ homopolymers, respectively. We study the elastic properties of the interface up to the point where the interface is saturated with copolymers, i.e., the interface tension $\sigma$ vanishes. Typically, segregation of amphiphiles to an interface can reduce its tension by several orders of magnitude, before the interface becomes unstable and the amphiphiles segregate to form a microphase with periodic order or a microemulsion. Hence, the saturation point gives a good, but slightly too large, estimate of how many copolymers an interface can accommodate [30].

The self-consistent field (SCF) theory neglects fluctuations. They are controlled by the degree $\bar{N}$ of interdigitation. The chain length $N$ enters the SCF calculations only in the combination $\chi N$, which sets the temperature scale, and $R_{e}$, which determines the length scale. If physical systems have identical $\chi N$ and $R_{e}$ but different density $\rho$ or chain length $N$ (and therefore different $\bar{N}$ ), the SCF theory will make identical predictions. Fluctuations of the local interface position (i.e., capillary waves) modify interface properties. Most notably, the width of the interface $w$ and the bending elastic constants $K$ and $\bar{K}$ depend on the lateral length scale $\xi_{\|}$, on which they are measured. Expressing the quantities in the dimensionless units of the SCF calculations, we obtain

$$
\begin{gathered}
\frac{w^{2}}{R_{e}^{2}}=\frac{w_{\mathrm{SCF}}^{2}}{R_{e}^{2}}\left(1+\frac{f_{w}\left(\chi N, R_{e}, \ldots\right) k_{B} T R_{e}^{2}}{4 \pi \sqrt{\bar{N}} w_{\mathrm{SCF}}^{2}} \ln \left[\frac{\xi_{\|}}{B}\right]\right), \\
K=K_{\mathrm{SCF}}\left(1+\frac{f_{K}\left(\chi N, R_{e}, \ldots\right) k_{B} T}{4 \pi \sqrt{\bar{N}} K_{\mathrm{SCF}}} \ln \left[\frac{\xi_{\|}}{B}\right]\right), \\
\bar{K}=\bar{K}_{\mathrm{SCF}}\left(1+\frac{f_{\bar{K}}\left(\chi N, R_{e}, \ldots\right) k_{B} T}{4 \pi \sqrt{\bar{N}} \bar{K}_{\mathrm{SCF}}} \ln \left[\frac{\xi_{\|}}{B}\right]\right),
\end{gathered}
$$

where $f_{w}, f_{K}$, and $f_{\bar{K}}$ are functions that depend on the parameters of the SCF calculations but not explicitly on $\bar{N}, f_{w}=\pi \Sigma$. For saturated interfaces $f_{K}$ and $f_{\bar{K}}$ take the values -3 and $10 / 3 . B$ is the lateral length scale on which the width and the bending rigidities adopt their intrinsic value. Calculations [31] and Monte Carlo simulations [32] of homopolymer interfaces suggest $B \approx \pi w_{\mathrm{SCF}}$. There is also a renormalization of the interface tension $\Sigma$, which is of the order $\ln [\xi]] / \xi^{2}$. In this sense, $\bar{N}$ controls the effect of fluctuations and the SCF calculations become quantitatively accurate in the limit $\bar{N} \rightarrow \infty$. In principle, polymers offer the opportunity to study the effect of fluctuations on interface properties in great detail by varying the degree of interdigitation. We would like to emphasis, however, that for typical experimental value of $\bar{N}$ fluctuation effects are important. For example, the additional broadening of the observed interface width in experiments due to capillary waves is of the same order of magnitude as the intrinsic width which is predicted by the SCF theory.

\section{RESULTS}

\section{A. Behavior of the elastic properties at a tricritical Lifshitz or isotropic Lifshitz point}

When a sufficient amount of a symmetric copolymer is added to a mixture of two homopolymers of identical length $\left(\alpha_{1}=1\right)$, the blend can be made compatible provided the incompatibility is low enough $(\chi N<6)$. At $\bar{\phi}_{1}=1-2 / \chi N$ one encounters a critical point (Scott line), where the difference between the two coexisting homopolymer-rich phases vanishes, the excess of copolymer approaches zero, and the width of the interface diverges. At larger segregation, the homopolymer-rich phases can accommodate only a small amount of copolymers. Adding more copolymer to the system leads to the formation of a lamellar phase. The two qualitatively different behaviors are separated by a Lifshitz tricritical point at $\chi N=6, \bar{\phi}_{1}=2 / 3$, and $z=4$ [1]. The excess $\Omega$ of copolymers as a function of the copolymer concentration $\bar{\phi}_{1}$ in the bulk is shown in Fig. 2(a).

In Figs. 2(b) and 2(c), we present the bending rigidity $K$ and the saddle-splay modulus $\bar{K}$, respectively, as a function of the excess of the copolymers at the interface. Each line is obtained by varying the block copolymer concentration in the bulk. The bending rigidity $K$ of a homopolymer interface (i.e., $\Omega=0$ ) is negative [18] and vanishes both at the critical point $\chi N=2$ and in the strong segregation limit $\chi N \rightarrow \infty$ [20]. The different curves correspond to different incompatibilities $\chi N$. For $\chi N<6$ they bend back and end at vanishing elastic constants, because the interface disappears as we add sufficient copolymers. For larger $\chi N$ the curves end at saturation $(\Sigma=0)$, but there is still a well-defined interface between $A$-rich and $B$-rich domains, and the absolute value of the elastic constants is maximal for that temperature. This maximal value of the elastic constant at saturation, i.e., the end point of the curves for $\chi N>6$, is depicted as a thick dashed line. In the wet brush regime $\Omega \ll 1$ the value of the elastic constant is very small, but for $\Omega \gg 1$ (dry brush) they 


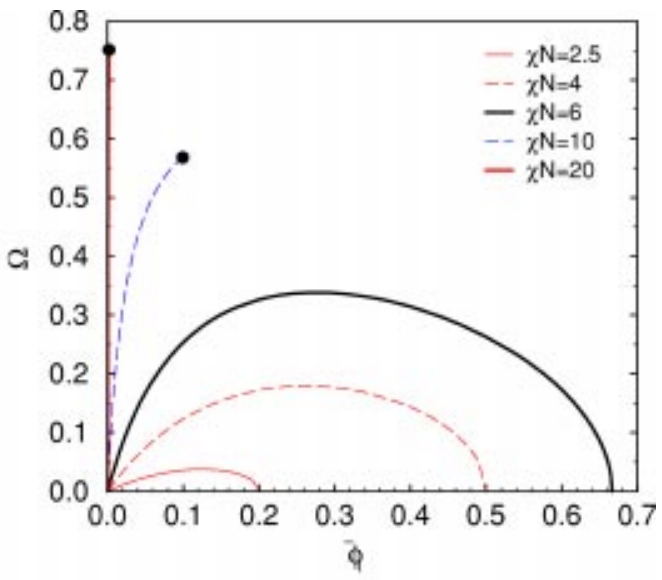

(a)

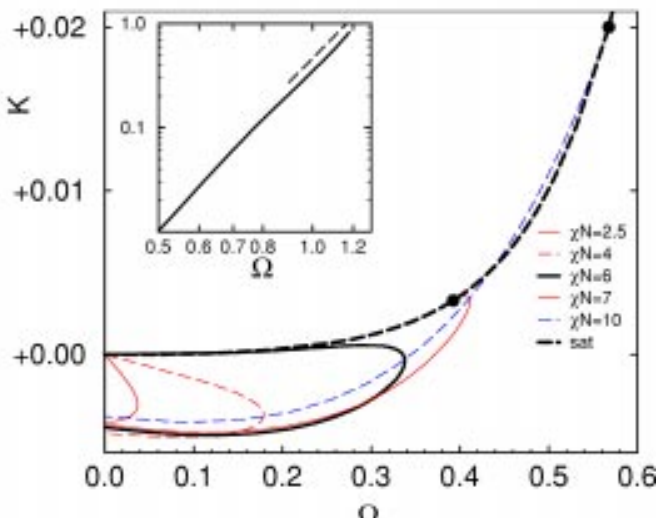

(b)

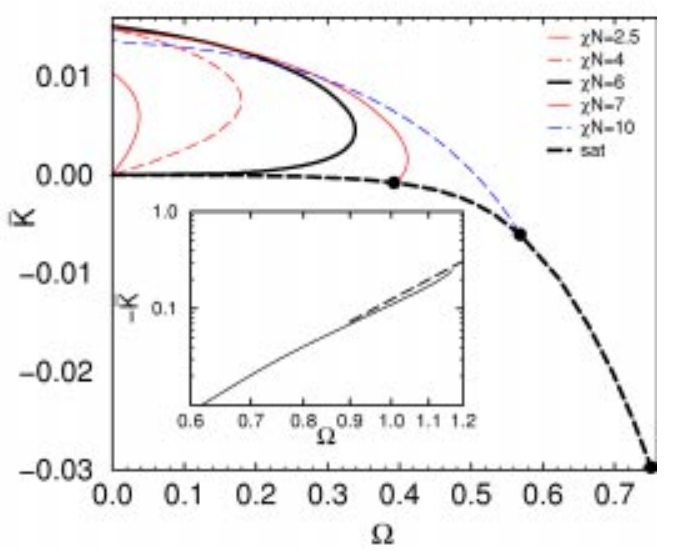

(c)

FIG. 2. Interface excess $\Omega$ as a function of the bulk concentration $\bar{\phi}_{1}$ of copolymers for various $\chi N$. Bending rigidity $K(\mathrm{~b})$ and saddle-splay modulus $\bar{K}$ (c) as a function of the excess of copolymers $\Omega$ at the interface. The chain length of the homopolymers equals that of the copolymers $(\alpha=1)$. The different curves correspond to different incompatibilities $\chi N$. For $\chi N<6$ the curves loop back and end at the origin. The thick line depicts the values of the elastic constants at saturation for $\chi N>6$. The saturation values for $\chi N=7$ and 10 are marked by filled circles. The inset shows the dependence of the saturation value on the interface excess. The dashed lines represent the predictions in the strong segregation limit.

steeply increase with the excess of copolymers. In the strong segregation limit we expect $K=\left(3 \pi^{2} / 64\right) \Omega^{5}$ and $\bar{K}=$ $-\left(\pi^{2} / 80\right) \Omega^{5}[18,33]$. These power laws are depicted in the inset of Fig. 2. The crossover between the wet and dry brush behavior has been investigated by Matsen [18] at constant $\chi N$. Our finding at saturation confirm his finding that only for extremely large segregation $\chi N \sim O\left(10^{3}\right)$ the predictions of the strong stretching assumption become quantitatively accurate.

In the vicinity of the Lifshitz tricritical point at $\chi N=6$, fluctuations are particularly important. Their relevance on the homopolymer-rich phases can be gauged by the Ginzburg criterium [34]: fluctuations are negligible, if the composition of the uniform homopolymer-rich phases in a volume of the size of the correlation length exhibits bulklike fluctuations which are small compared to the difference in composition between the coexisting homopolymer-rich phases. Using the appropriate critical exponents for the tricritical Lifshitz universality class, Holyst and Schick [2] calculated that $|\chi N-6| \gg \bar{N}^{-2 / 3}$ is required for the mean-field theory to hold.

Indeed, Monte Carlo simulations [4] and experiments $[5,6,9,7]$ do not confirm the mean-field prediction of a highly swollen (asymmetric) lamellar phase [1-3] in the vicinity of the tricritical Lifshitz point, but rather find a disordered microemulsionlike state. In the vicinity of a Lifshitz point the interface between $A$-rich and $B$-rich regions is very wide and the interface tension is very small. It is the bending rigidity $\kappa$ which stabilizes the lamellar phase against fluctuations of the orientation of the interfaces. de Gennes and Taupin [35] calculated the correlation length of the direction of an isolated interface to be $\xi \sim R_{e} \exp \left(2 \pi \kappa / k_{B} T\right)$. For $\kappa \sim k_{B} T$ the correlation length of the normal direction of the lamellae becomes small, fluctuations of the local interface position destroy the long-range lamellar order, and a microemulsion is formed instead. In fact, a more detailed analysis [16,36,37] shows that for sufficiently large bending rigidity, the transition from the lamellar phase to the microemulsion phase is controlled by the saddle-splay modulus $\bar{\kappa}$. When $|\bar{\kappa}| \gg k_{B} T$, the lamellar phase is stable when the interfaces are packed close together, but melts into a microemulsion phase at higher dilution [38].

In Fig. 3 we present how various quantities vary as the tricritical Lifshitz point is approached by changing the temperature $t=\chi N-6$ at fixed chemical potential [see Fig. 3(a)] or by increasing the copolymer's chemical potential $t=z$ -4 at fixed temperature [see Fig. 3(b)]. Our SCF calculations yield the following estimates for the mean-field exponents

$$
\begin{gathered}
\frac{1}{2}-\bar{\phi}_{A} \sim t^{\beta_{\text {tri }}} \quad \text { with } \quad \beta_{\mathrm{tri}}=1 / 4 \\
\Sigma \sim t^{\mu} \quad \text { with } \quad \mu=\left(d_{u}-1\right) \nu=(6-1) / 4=5 / 4 \\
\frac{2}{3}-\bar{\phi}_{1} \sim t^{1 / 2} \\
\Omega \sim t^{1 / 4} \\
K \sim \bar{K} \sim t^{3 / 4}
\end{gathered}
$$



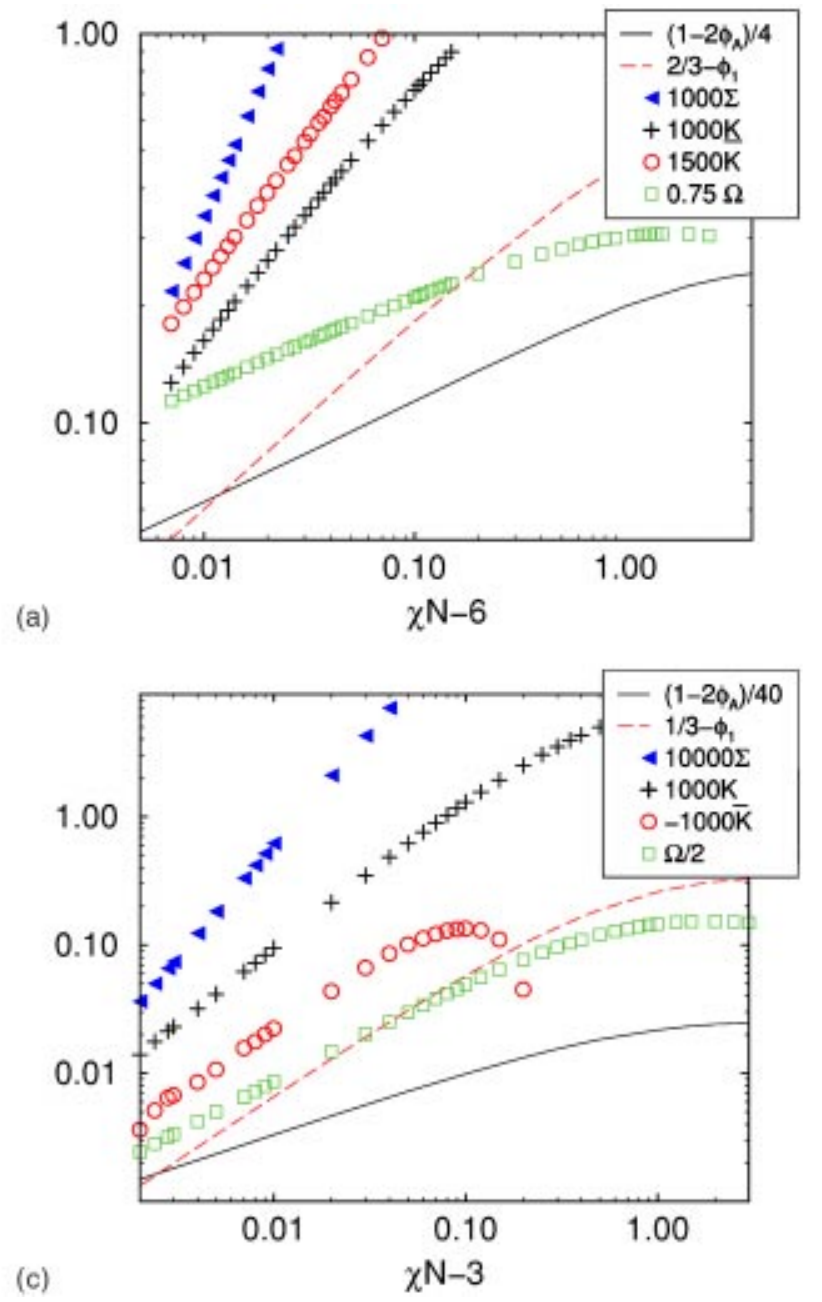

(b)
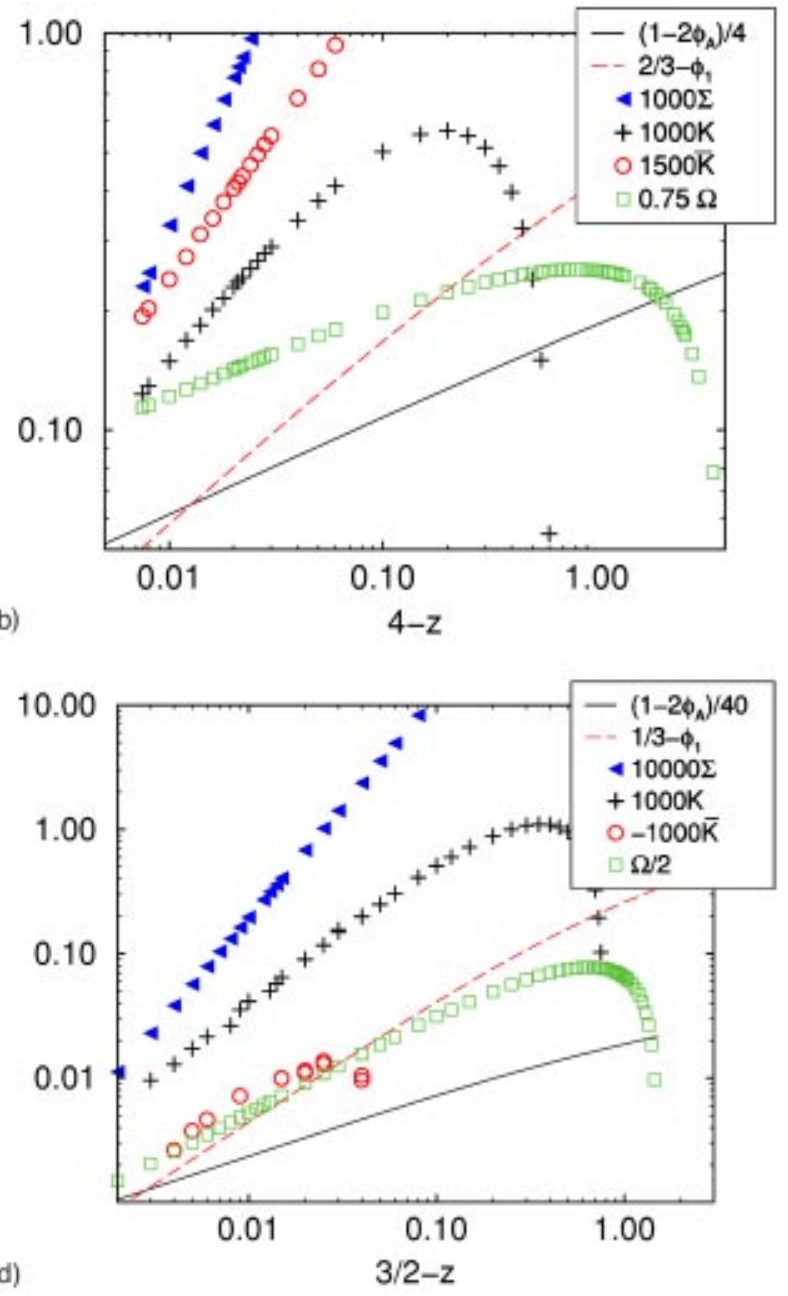

FIG. 3. Power law dependence of the composition, the bulk copolymer density, the interface tension, the bending rigidity, and the saddle-splay modulus upon approaching the Lifshitz tricritical point (a) and (b) for $\alpha=1$ and the isotropic Lifshitz point (c) and (d) for $\alpha=0.5$. From the slopes we estimate the critical exponents.

The first two exponents have been obtained previously from the general properties of a tricritical Lifshitz point [2]. The exponents of the bending and saddle-splay moduli have the same scaling dimension as $\Sigma \xi^{2}$ [39]. Requiring the bending rigidity to be at least of the order of $k_{B} T$ for the lamellar phase to be stable, we obtain $\kappa \sim \sqrt{\bar{N}} t^{3 / 4} \sim \mathcal{O}(1)$, i.e., $t$ $\gg \bar{N}^{-2 / 3}$. Closer to the Lifshitz tricritical point, fluctuations will disorder the lamellar phases predicted by the SCF theory into a microemulsion. Remarkably, consideration of interface fluctuations yield the same Ginzburg criterium as the consideration of composition fluctuations in the bulk.

If the copolymers are longer than the homopolymers $\left(\alpha_{1}<1\right)$, an isotropic Lifshitz critical point is formed [1]. In this case, the location of the Lifshitz critical point is a function of chain length, $\chi N_{\text {lif }}=2\left(1+2 \alpha_{1}^{2}\right)$ and $\phi_{1, \text { lif }}=2 \alpha_{1}^{2} /(1$ $+2 \alpha_{1}^{2}$ ) [1]. The comparison of the composition fluctuation in the bulk with the segregation of the coexisting phases, then implies $t \gg \bar{N}^{-2 / 5}$ as a Ginzburg criterium. Our SCF calculations for $\alpha=0.5$ are presented in Figs. 3(c) and 3(d). They yield

$$
\begin{gathered}
\frac{1}{2}-\bar{\phi}_{A} \sim t^{\beta} \quad \text { with } \quad \beta=1 / 2 \\
\Sigma \sim t^{\mu} \quad \text { with } \quad \mu=\left(d_{u}-1\right) \nu=(8-1) / 4=7 / 4 \\
\\
\frac{1}{3}-\bar{\phi}_{1} \sim t^{1} \\
\Omega \sim t^{3 / 4} \\
K \sim \bar{K} \sim t^{5 / 4}
\end{gathered}
$$

with $t=\chi N-3$ in Fig. 3(c) and $t=3 / 2-z$ in Fig. 3(d). The same reasoning as above now leads to $\kappa \sim \sqrt{\bar{N}} t^{5 / 4} \sim O(1)$ and $t \gg \bar{N}^{-2 / 5}$. Again, the latter criterium for the interfaces to be stable with respect to interface fluctuations is compatible with the Ginzburg criterium for bulklike composition fluctuations [2]. 


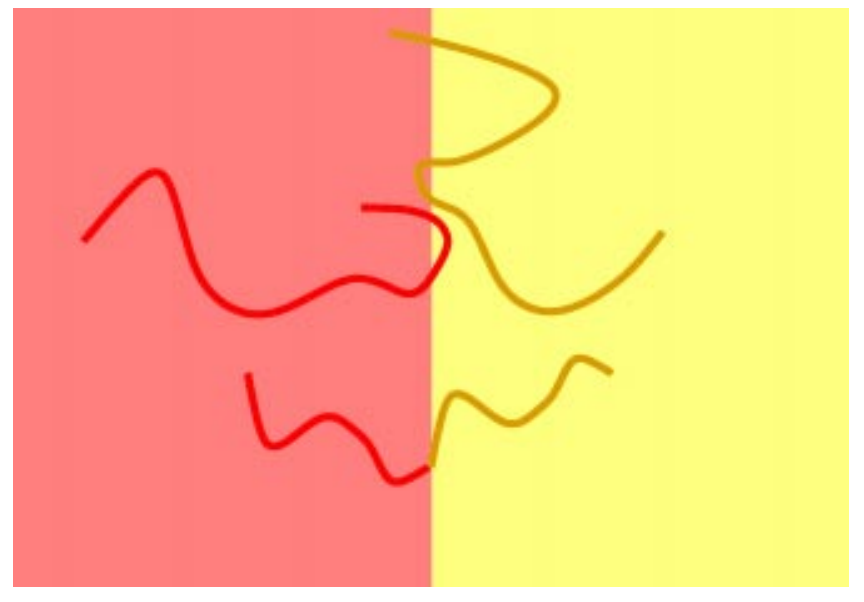

FIG. 4. Sketch of the typical conformations of homopolymers and diblock copolymers in the vicinity of the interface. Adding diblock copolymers and replacing some of the homopolymers at the interface by shorter ones have qualitatively similar effects.

\section{B. Small interfacial excess of symmetric diblock copolymers}

Let us now turn from saturated interfaces to the opposite limit of a vanishingly small amount $(\Omega \ll 1)$ of copolymers adsorbed to an interface and its influence on the elastic properties. The case of equal chain lengths $\left(\alpha_{1}=1\right)$ of diblock copolymers and homopolymers can be read off from Fig. 2. Except for the vicinity of the critical point $\chi_{c} N=2$ adsorption of a small amount of copolymers to an interface decreases the bending rigidity $K$; only at larger $\Omega$ the rigidity increases with the adsorbed amount. This somewhat surprising, nonmonotonic dependence is qualitatively similar to recent computer simulations of surfactants at a liquid-liquid interface by Laradji and Mouritsen [40]. They attribute this effect to a coupling between fluctuations of the interface position and the surfactant orientation. In the case of an interface between two homopolymers (cf. Fig. 4) we can qualitatively rationalize the effect as follows. Due to the incompressibility, each copolymer that adsorbs to the interface replaces homopolymers on each side of the interface. Thus a small amount of copolymers has a similar effect as replacing some of the homopolymers at the interface by shorter homopolymers-namely, the blocks of the copolymer. Replacing longer chains at the interface by shorter ones decreases the bending rigidity for sufficiently large $\chi N$ because $K$ increases with $N$ at constant Flory-Huggins parameter $\chi$. The opposite holds true in the vicinity of the critical point: here $K$ decreases with $\chi N$ and so the bending rigidity increases as we add a small amount of copolymers.

Due to the replacement of homopolymers, the behavior of short copolymers is opposed to the expectation for Gaussian chains tethered to an infinitely thin impenetrable membrane. In the latter case $\kappa$ increases and $\bar{\kappa}$ decreases upon adsorption of polymers. Hiergeist and Lipowsky [21], Eisenriegler and co-workers [22], and Marques and Fournier [23] have calculated the loss of conformational entropy $\Delta S$ for an isolated Gaussian chain of end-to-end distance $R_{p}$ and its dependence on the curvature,

$$
-T \Delta S=2\left(k_{B} T c R_{p}^{2}\right) H^{2}+\left(k_{B} T \bar{c} R_{p}^{2}\right) G
$$

where

$$
c=(1+\pi / 2) / 12 \approx 0.214 \ldots, \quad \bar{c}=-1 / 6 \approx 0.166 \ldots,
$$

for bending and saddle-splay deformations, respectively. When the excess $\Omega$ of copolymers at the interface is fixed and the copolymers do not interact (i.e., wet brush $\Omega \ll 1$ ), the contributions of the individual blocks are additive and lead to

$$
K=K(\Omega=0)+c \Omega \text { and } \bar{K}=\bar{K}(\Omega=0)+\bar{c} \Omega .
$$

We want to remark parenthetically that when the effect of copolymer chain length $N / \alpha_{1}$ is investigated at constant $\Omega$ in Eq. (35), the number density of copolymers at the interface is not constant, but decreases with decreasing $\alpha_{1}$. Therefore, the brush remains in the wet state even for $\alpha_{1} \rightarrow 0$.

Our calculations are performed in the grand-canonical ensemble, i.e., we do not hold the interface excess constant but rather fix the chemical potential of the copolymers. The excess of the copolymers is in chemical equilibrium with the bulk phase at fugacity $z$. The free energy of the copolymer adsorbed at the interface comprises two contributions: the translational entropy of the junction point, which is confined to the interface and the conformational entropy loss due to the confinement of the blocks in the appropriate phases. The latter contribution is given by Eq. (33). For $\Omega \ll 1$ the diblocks do not interact and, therefore, we neglect any contribution due to chain stretching. Then, the fugacity of the diblock copolymers at the interface is given by

$$
\begin{aligned}
\ln z= & \ln \left(\frac{\Omega(z, H, G) R_{e} \alpha}{w}\right)+2\left(k_{B} T c R_{e}^{2} / \alpha\right) H^{2} \\
& +\left(k_{B} T \bar{c} R_{e}^{2} / \alpha\right) G
\end{aligned}
$$

where $w$ is the width of the profile of junction points. As we increase the curvature of the interface, the excess $\Omega(z, H, G)$ has to decreases as to keep the fugacity at the constant bulk value. The change of the free energy due to the desorption of copolymers can be calculated via the Gibbs-Duhem adsorption isotherm. Using Eq. (36), we obtain

$$
\begin{aligned}
\Delta g & \equiv g(z)-g(z=0) \\
= & -\int_{0}^{z} \frac{d z^{\prime}}{z^{\prime}} \Omega\left(z^{\prime}, H, G\right) \alpha \\
= & -\Omega(z, H, G) \alpha \\
\approx & -\Omega \alpha+\Omega\left(2 c R_{e}^{2} H^{2}+\bar{c} R_{e}^{2} G\right) \\
& \text { with } \quad \Omega \equiv \Omega(z, H=0, G=0) .
\end{aligned}
$$

In the wet brush limit, each copolymer at the interface lowers the free energy by $k_{B} T$. Bending the interface gives rise to a desorption of the copolymers and their contribution to the interface free energy $\Delta g$ increases. The incremental change 
of the elastic constants at chemical equilibrium is identical to the increment at constant interface excess (35).

The SCF results for $c=d K / d \Omega$ and $\bar{c}=d \bar{K} / d \Omega$ at $\chi N$ $=20$ and various length of the copolymer are shown in the insets of Fig. 5. Upon increasing the chain length of the copolymer, the values approach the prediction for Gaussian chains at an impenetrable surface very gradually from below. Even if the copolymers are a factor 100 longer than the homopolymers, $c$ and $\bar{c}$ differ by more than $40 \%$ from the limiting values of polymers tethered to a structureless sheet. These deviations highlight the importance of the interface structure: there is a strong interplay between the copolymer and the other constituents of the interface.

\section{Two-component mixtures of symmetric diblock copolymers at interfaces}

In recent experiments $[10,11,41]$ on polyethylenepropylene-polyethyleneoxide (PEP-PEO) diblock copolymers at an oil-surfactant-water interface, already a small amount of block copolymer was found to strongly reduce the amount of short-chain surfactant required to form a bicontinuous microemulsion. This effect has been attributed to the ability of the copolymer to make the saddle-splay modulus $\bar{\kappa}$ more negative [11]. However, it is not possible to obtain a microemulsion of water, oil, and a long-chain block copolymer, because the interface excess remains small, and the polymer rather forms micelles at higher concentrations. Thus, the role of the surfactant is to reduce the interfacial tension to ultralow values.

We want to investigate here the same mechanism in polymer blends. Therefore, we consider how a small amount of long copolymer $\left(\alpha_{2}\right)$ affects the elastic constants of an interface between two immiscible homopolymers saturated with a short diblock copolymer $1 / \alpha_{1}=0.6$. In Fig. 5 we show the change of the elastic constant upon adsorption of a vanishingly small amount of long copolymer. The behavior of the bending rigidity and the saddle-splay modulus is qualitatively very similar to the effect of a single diblock copolymer at the interface, as described in the preceding paragraph. In the limit of very long copolymers $\alpha_{2} \rightarrow 0$, our data are compatible with the asymptotic behavior of polymers tethered to a structureless membrane. For finite chains lengths, however, the effect of the long copolymer is overestimated by the structureless membrane model.

The dependence of the interface excess on the curvature for a cylindrical deformation is depicted in Fig. 6. Upon bending, both copolymer species desorb and the interface excess depends quadratically on the dimensionless curvature $C=R_{e} / R$ as expected for an interface without spontaneous curvature.

The deviations from the structureless interface model can be attributed in part to the competition between short and long chains at the interface. As the long chains adsorb, they expel the short surfactants from the interface. So the effect of the long copolymer alone is rather
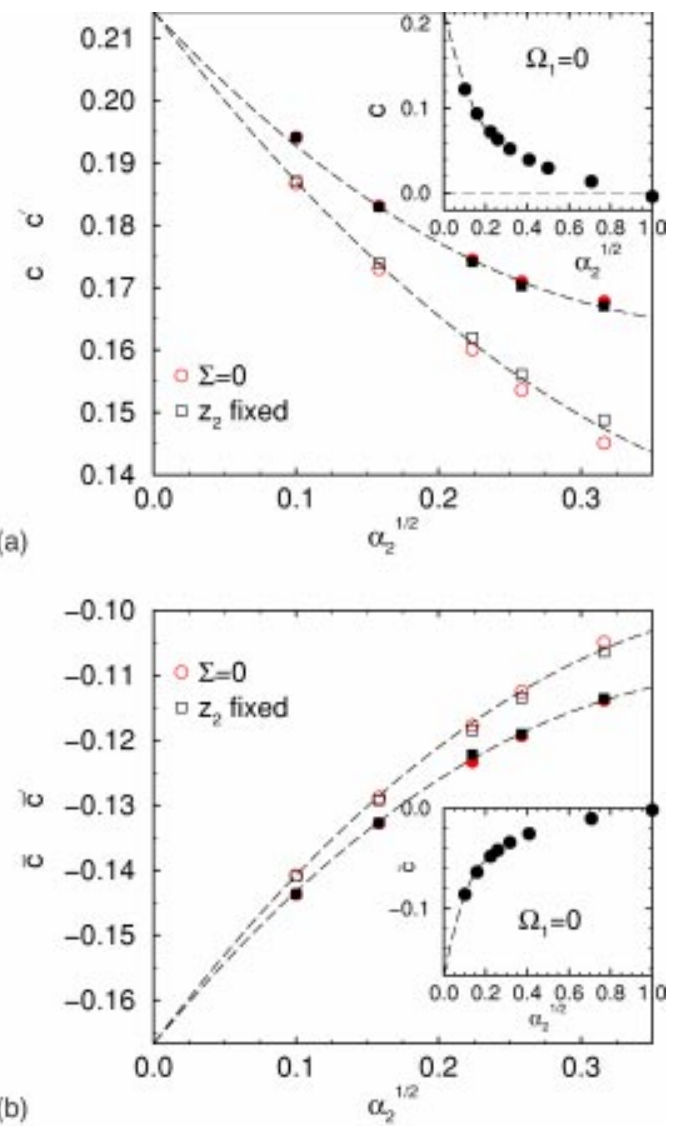

FIG. 5. Change of the elastic constants [(a) bending rigidity and (b) saddle-splay modulus] upon adsorption of a small amount of diblock copolymers with chain length $1 / \alpha_{2}$ (compared to the homopolymers). The interface at $\chi N=20$ is saturated with shorter diblock copolymers of relative length $1 / \alpha_{1}=0.6$. Circles correspond strictly to saturation, i.e., $\Sigma=0$ after the adsorption of copolymers. Squares correspond to an adsorption at a fixed fugacity, chosen such that the interface without the adsorption of the long diblock copolymers is saturated. Open symbols refer to elastic constants of the interface, filled circles subtract the contribution due to the desorption of the shorter diblock copolymers. Lines are guides to the eye. The inset presents the data for an interface between two homopolymers without shorter diblock copolymers at $\chi N=20$.

$$
\begin{aligned}
& c^{\prime}=\frac{d K}{d \Omega_{2}}-\frac{d K}{d \Omega_{1}} \frac{d \Omega_{1}}{d \Omega_{2}}, \\
& \bar{c}^{\prime}=\frac{d \bar{K}}{d \Omega_{2}}-\frac{d \bar{K}}{d \Omega_{1}} \frac{d \Omega_{1}}{d \Omega_{2}} .
\end{aligned}
$$

In order to observe the prediction of the structureless interface model at least in the limit of long copolymers $\alpha_{2}$ $\rightarrow 0, d \Omega_{1} / d \Omega_{2}$ has to vanish. A wet and a dry brush of short surfactants can be distinguished.

(i) $\Omega_{1} \ll 1$. If we fix the chemical potentials of the copolymers, $\Omega_{1}$ will not depend on the excess of the long copolymers. If the interface is saturated $\Sigma_{0}-\Omega_{1} \alpha_{1}-\Omega_{2} \alpha_{2}=0$, and the derivative will take to form $d \Omega_{1} / d \Omega_{2}=-\alpha_{2} / \alpha_{1}$.

(ii) $\Omega_{1}>1$. If we fix the the chemical potentials, each long chain approximately replaces one short surfactant, and 


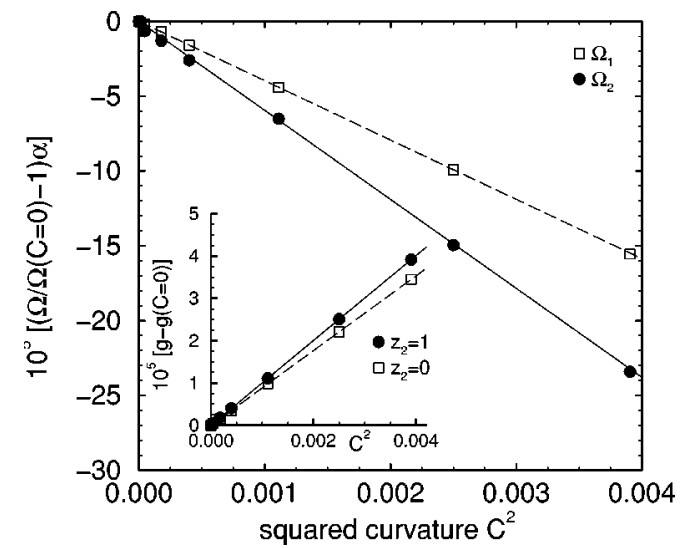

FIG. 6. Dependence of the adsorbed amount of copolymers on the curvature for a cylindrical deformation. The fugacity of the short copolymer $\alpha_{1}=1 / 0.6$ corresponds to its saturation value $z_{1}$ $=21.316$ at $\chi N=20$. The chain length of the second copolymer is $1 / \alpha_{2}=20$ and its fugacity $z_{2}=1$. Both copolymers leave the interface as the curvature is increased. The dashed and solid lines in the main panel correspond to $\alpha[\Omega / \Omega(C=0)-1] \times 10^{5}=-0.079 C^{2} / 2$ and $-0.119 C^{2} / 2$, respectively. The inset presents the free energy as a function of curvature with $\left(z_{2}=1\right)$ and without $\left(z_{2}=0\right)$ long copolymers. From the difference of the slopes we estimate the increase of $\kappa$ due to the adsorption of a small amount of large copolymers. The dashed and solid lines correspond to $[g-g(C$ $=0)] \times 10^{5}=0.01767 C^{2} / 2$ and $0.02007 C^{2} / 2$, respectively.

we will observe $d \Omega_{1} / d \Omega_{2}=-\alpha_{2} / \alpha_{1}$. If we require the interface to be saturated, the adsorption isotherm will yield $\Sigma_{0}-W\left(\Omega_{1} \alpha_{1}\right)-\Omega_{2} \alpha_{2}=0$, where $W$ is an increasing function of its argument. This expression results in $d \Omega_{1} / d \Omega_{2}$ $=-\alpha_{2} /\left[W^{\prime}\left(\Omega_{1} \alpha_{1}\right)\right]$. In any case the last term in Eq. (38) is only of the order $\alpha_{2}$ and we expect to recover the prediction of the structureless interface model for $\alpha_{2} \rightarrow 0$.

The values $c^{\prime}$ and $\bar{c}^{\prime}$ are also depicted in Fig. 5. The correction reduces the deviation from the structureless interface model, but cannot account for our data quantitatively. The graph suggests that the leading correction is of order $\sqrt{\alpha_{2}}$ while the correction above is only of the order $\alpha_{2}$. Additionally, the finite thickness of the interface and the nonadditive dependence [42] of the elastic constants on the adsorbed amounts of the two surfactants have to be considered.

\section{Mixtures of two asymmetric diblock copolymers at interfaces: Importance of flip/flop}

In this subsection we investigate the role of adsorption or desorption of asymmetric amphiphiles on the bending rigidity. We choose a system with two asymmetric diblock copolymers $f_{1}=2 / 2.3$ and $f_{2}=1-f_{1}$ of length $\alpha_{1}=\alpha_{2}$ $=1 / 2.3$. Each amphiphile imparts a spontaneous curvature onto the interface. When they adsorb in equal amounts to the interface, however, the interface is symmetric. In Fig. 7(a) we present the segment profiles at saturation. The profiles (2asym) are compared to profiles across an interface with two symmetric copolymers $f_{1}=f_{2}=1 / 2$ of length $1 / \alpha_{1}$ $=0.6$ and $1 / \alpha_{2}=4$ at saturation (2sym) and profiles of an interface at which the same amount of only one asymmetric

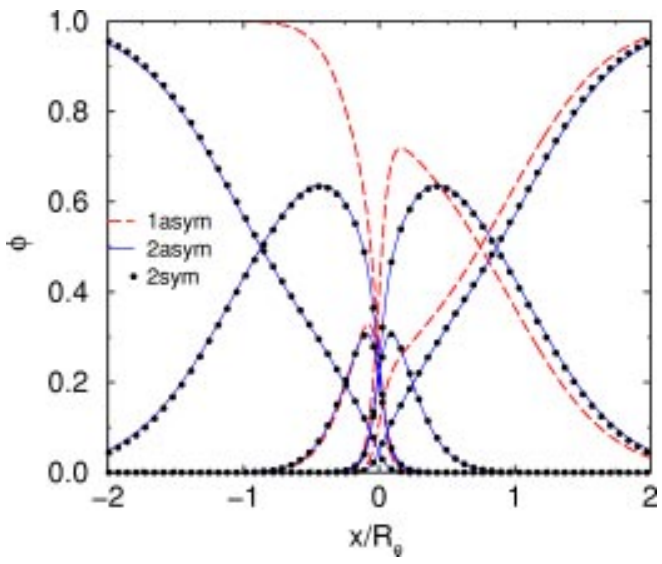

FIG. 7. Density profiles of the homopolymers and diblock copolymers across the interface at $\chi N=36$ for the three systems compared in the text and Table I. 1asym refers to an interface with a single asymmetric copolymer, 2asym denotes the excess of an interface with two asymmetric copolymers adsorbed to it, and 2sym labels the data for an interface with two symmetric copolymers adsorbed. The latter two profiles have very similar shape, excess, and both correspond to saturation.

diblock (1asym) is adsorbed. The latter profiles differ somewhat from the case of two asymmetric copolymers: the $A B$ interface is sharper, the interface tension is positive, and the long block extends less into the bulk regions. The profiles for the adsorption of two asymmetric (2asym) and two symmetric $(2$ sym) copolymers are very similar: it requires the same amount of copolymers to saturate the interface $(\sigma=0)$ and the profiles of the individual blocks are almost identical. Additionally, the saddle-splay modulus between both cases differs by less than $1 \%$. There is, however, a marked difference in the bending rigidity. In the case of two asymmetric copolymers $K$ is a factor 3.4 smaller than for the symmetric case. The elastic constants and other data are compiled in Table I.

This difference stems from selective adsorption or desorption of the asymmetric copolymer upon increasing the cur-

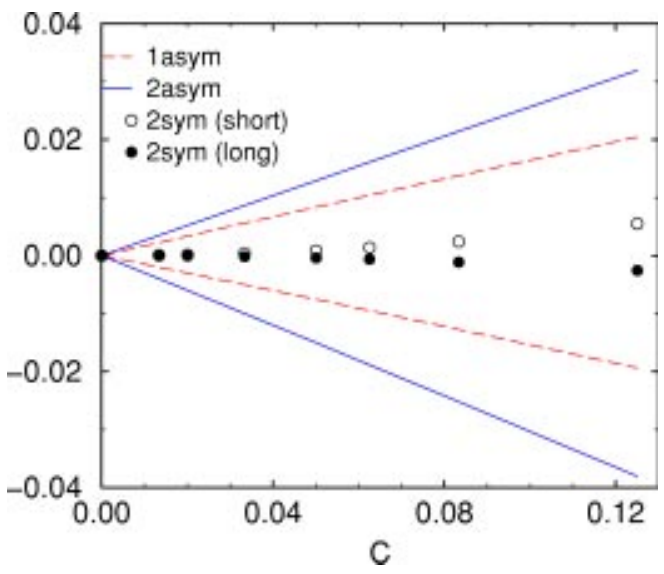

FIG. 8. Dependence of the interface excess $\Omega(C)-\Omega(C=0)$ on the curvature for a cylindrical deformation. 1asym, 2asym, and 2 sym refer to the systems discussed in the text (cf. Fig. 7). The two curves for a single asymmetric diblock correspond to different signs of the curvature. 
TABLE I. Interface excess and elastic constants for the three situations compared in the text.

\begin{tabular}{|c|c|c|c|}
\hline & 1asym & 2asym & $2 \mathrm{sym}$ \\
\hline $1 / \alpha_{1}$ & 2.3 & 2.3 & 0.6 \\
\hline $1 / \alpha_{2}$ & - & 2.3 & 4.0 \\
\hline$z_{1}$ & 46.07 & 164.74 & 18.73 \\
\hline$z_{2}$ & 0 & 164.74 & 1425.22 \\
\hline$\Omega_{1} \alpha_{1}$ & 0.382 & 0.382 & 0.386 \\
\hline$\Omega_{2} \alpha_{2}$ & 0 & 0.382 & 0.386 \\
\hline$\Sigma$ & 1.412 & 0 & 0 \\
\hline$\Lambda$ & -0.49 & 0 & 0 \\
\hline$K$ & 0.124 & 0.312 & 1.074 \\
\hline$\overline{\boldsymbol{K}}$ & -0.149 & -0.506 & -0.503 \\
\hline
\end{tabular}

vature. The dependence of the interface excess on the curvature for a cylindrical deformation is presented in Fig. 8. As the curvature $C$ increases, one of the asymmetric species adsorbs to the interface and the other desorbs. The change in the excess depends linearly on the curvature $C$. The effect is even stronger than for a single asymmetric copolymer at an interface. This behavior of the asymmetric copolymers contrasts with that for symmetric ones. In the latter case (2sym) the short diblock copolymers adsorb and the longer ones desorb from the interface. The magnitude of the effect is much smaller and it depends only quadratically on the curvature.

We compare our results to a phenomenological description [24-27]. The interfacial free energy comprises two contributions: a term that describes the free energy of mixing of the two species of amphiphiles and the effective interface Hamiltonian (1),

$$
\mathcal{H}[\phi]=\int d S\left[\frac{1}{2} \tau \phi^{2}+\left\{\sigma+\lambda(\phi) H+2 \kappa_{0} H^{2}+\bar{\kappa} G\right\}\right],
$$

where $\phi=\left(\Omega_{1}-\Omega_{2}\right) /\left[2\left(\Omega_{1}+\Omega_{2}\right)\right]$ denotes the composition of the interface. $\tau$ measures the free energy cost of deviations from symmetric composition. The spontaneous curvature $\lambda$ depends on the composition; it vanishes only for the symmetric case $\phi=0$. For nearly symmetric composition (i.e., small curvatures) it takes the form $\lambda(\phi)=\gamma \phi$. Minimizing the effective interface Hamiltonian with respect to the composition of the interface, we obtain $\phi^{*}=-\gamma / \tau H$ and

$$
\mathcal{H}\left[\phi^{*}\right]=\int d S\left\{\sigma+2 \kappa_{0}\left[1-\frac{\gamma^{2}}{4 \kappa_{0} \tau}\right] H^{2}+\bar{\kappa} G\right\} .
$$

The adjustment of composition $\phi^{*}$ on the curvature $H$ reduces the effective bending rigidity by a factor 1 $-\gamma^{2} /\left(4 \kappa_{0} \tau\right)$, but leaves the saddle-splay modulus unaltered. This is an agreement with our calculations.

To compare this simple phenomenological description quantitatively to our SCF calculations, we rewrite

$$
\Lambda=\Gamma \phi \text { with } \Gamma=\frac{\gamma R_{e}}{\sqrt{\bar{N}} k_{B} T},
$$

$$
\begin{gathered}
\phi^{*}=-\frac{\Gamma C}{2 t} \quad \text { with } \quad t=\frac{\tau R_{e}^{2}}{\sqrt{\bar{N}} k_{B} T}, \\
K=K_{0}\left(1-\frac{\Gamma^{2}}{4 t K_{0}}\right) .
\end{gathered}
$$

The spontaneous curvature as a function of the composition of the mixed surfactant layer is presented in Fig. 9. From the slope at $\phi=0$ we determine $\Gamma=5.2$. We note that the linear relation between composition and spontaneous curvature holds only over a rather limited range of composition and higher-order terms in the phenomenological description would be required to describe the SCF results for all composition. The figure also shows the variation of the elastic constants $K$ and $\bar{K}$ on composition. Again, the nonadditivity

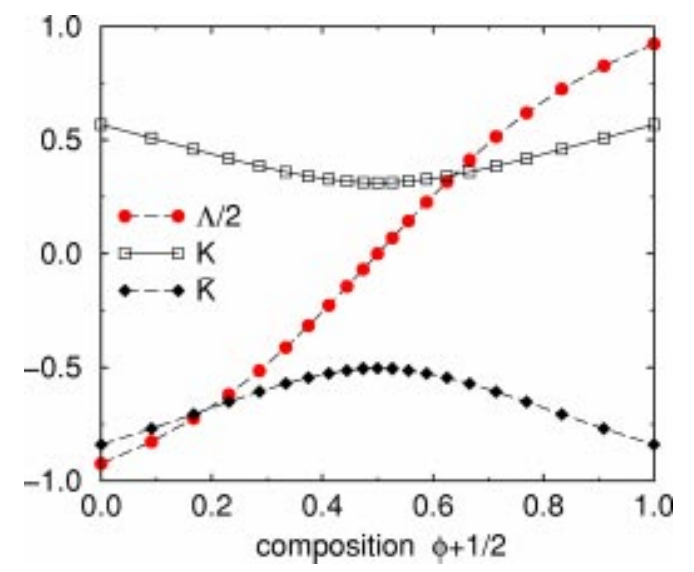

FIG. 9. Dependence of the elastic constants on the composition of the surfactant layer adsorbed to the interface at $\chi N=36$. The surfactant mixture comprises two diblock copolymer species of relative length $1 / \alpha_{1}=1 / \alpha_{2}=2.3$ and architecture $f_{1}=1-f_{2}$ $=0.3 / 2.3$. Symmetric composition $\phi=0$ corresponds to system 2asym. 
points to the omission of higher-order terms in Eq. (40). From the dependence of the composition of the mixed surfactant layer on the curvature (cf. Fig. 8) we estimate $t$ $\approx 8.7$. Using the bending rigidity of the interface with two symmetric copolymers $K_{0}=1.07$ the simple model predicts a reduction of the bending rigidity by a factor 3.7. This is in qualitative agreement with the reduction 3.4 observed in the SCF calculations. The deviation is presumable due to the uncertainty in the value of $K_{0}$ : on one hand the estimate from the interface with two symmetric copolymers is only approximate, on the other hand $K_{0}$ depends additionally on the composition $\phi$.

\section{E. Role of the surfactant architecture: $A B A$-triblock copolymers versus diblock copolymers}

Intriguingly, the structureless interface model predicts [43] that a Gaussian chain which is tethered with both ends to an impenetrable sheet does not impart a spontaneous curvature onto it. Due to the chain connectivity there is an effective attraction between the two anchoring points of the polymer on the sheet, which makes the sheet bend towards the polymer. Such a curvature, however, restricts the chain conformations, which, in turn, favors a bending of the sheet away from the polymer. Both effects cancel exactly.

Such a system may be realized by a symmetric $A B A$-triblock copolymer. The short outer $A$ blocks anchor the polymer at the interface between the immiscible homopolymers and the longer middle $B$ block corresponds to the Gaussian chain. We expect the prediction of the structureless interface model to be accurate if the extension of the middle block exceeds all other length scales (i.e., the width of the interface or the size of the anchoring block). If we kept the size of the anchoring block fixed, and simply increased the length of the middle block, unfortunately, the chains would not segregate to the interface but would rather stay in the $B$-rich phase [44]. Hence, we chose a fixed but small composition $f=1 / 10$. In the following we study the influence of a small amount of triblock copolymer on the elastic properties of an interface between two homopolymers and a short $\left(1 / \alpha_{1}=0.6\right)$ diblock copolymer with zero interface tension at $\chi N=20$.

In this case, we would expect that the spontaneous curvature is determined by the two anchoring blocks of length $N_{A}=f N / 2 \alpha_{\text {tri }}$. Within the approximations of the structureless interface model the contribution of a single block takes the form [21]

$$
\begin{gathered}
-T \Delta S=-c^{\prime} k_{B} T R_{p} H+O\left(H^{2}, G\right) \quad \text { with } \\
c^{\prime}=\sqrt{\pi / 6} \approx 0.724 \ldots,
\end{gathered}
$$

where $R_{p}$ denotes the end-to-end distance of the block. For the wet brush regime, we obtain the scaled spontaneous curvature

$$
\Lambda_{\mathrm{tri}}=-c^{\prime}\left(2 \Omega_{\mathrm{tri}}\right) \sqrt{f \alpha_{\mathrm{tri}} / 2} \approx-0.324 \Omega_{\mathrm{tri}} \sqrt{\alpha_{\mathrm{tri}}}
$$

by summing over the individual contributions of the $A$ blocks only. We want to compare this result with the case of diblock

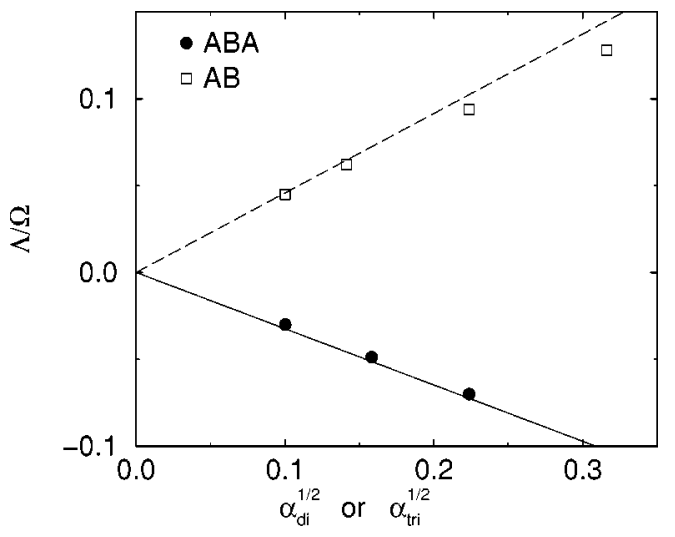

FIG. 10. Spontaneous curvature $\Lambda$ as a function of the chain length of $A B A$-triblock copolymers and asymmetric diblock copolymers of identical composition but half the length. $\chi N=20$ and the interface is saturated with short symmetric diblocks $1 / \alpha_{1}$ $=0.6$. The predictions (43) and (44) of the structureless interface model are indicated as lines.

copolymers, which are obtained by cutting the triblock into two identical pieces of half the length, so that $\alpha_{d i}=2 \alpha_{t r i}$ and $\Omega_{\mathrm{di}}=\Omega_{\text {tri }}$. The scaled spontaneous curvature in this case is predicted from the structureless interface model to be

$$
\Lambda_{\mathrm{di}}=-c^{\prime} \Omega_{\mathrm{di}}(\sqrt{1-f}-\sqrt{f}) \sqrt{\alpha_{\mathrm{di}}} \approx 2 \times 0.324 \Omega_{\mathrm{di}} \sqrt{\alpha_{\mathrm{di}} / 2},
$$

i.e., for this choice of composition $f=1 / 10$ cutting the triblock into two identical pieces changes the spontaneous curvature from $\Lambda_{\text {tri }}$ to $\Lambda_{\mathrm{di}}=-2 \Lambda_{\text {tri }}$.

In Fig. 10 we show the spontaneous curvature per surfactant $\Lambda / \Omega$ as a function of $\sqrt{\alpha_{\text {tri }}}$ or $\sqrt{\alpha_{\mathrm{di}}}$. Indeed, the middle block of the triblock copolymer does not contribute much to the spontaneous curvature and for long chains $\alpha \rightarrow 0$ the predictions of the structureless interface model become accurate, while there are deviation for small chain length, because the width of the interface and the block size are not well separated.

\section{SUMMARY AND DISCUSSION}

The addition of a small amount of surfactants to an interface can significantly modify its elastic properties. This effect has attracted abiding interest for the stabilization of bicontinuous microemulsions in polymer blends and oil-water mixtures. The elastic properties of the copolymer-laden interface are a key for predicting the stability region of the microemulsion. We investigate the elastic constants in the framework the SCF theory [18] for an interface between two homopolymers. Our calculations take due account of the local structure of the interface, and the results are compared with phenomenological predictions or calculations for a structureless, impenetrable interface.

The case of a symmetric diblock copolymer at an interface between two immiscible homopolymers has attracted much interest, because of the formation of a polymeric microemulsion $[4,5]$ in the vicinity of parameters where the mean-field theory predicts Lifshitz critical behavior. The 
breakdown of mean-field theory is anticipated by the Ginzburg criterium [2], which compares the magnitude of bulklike composition fluctuations with the width of the miscibility gap. Alternatively, it has been suggested that fluctuations destroy the orientational order of the highly swollen lamellae in the vicinity of a Lifshitz point and lead to a structure with no long-ranged order. Calculating how the elastic constants vanish upon approaching the Lifshitz or tricritical Lifshitz point, we show that both criteria yield the same predictions for the extension of the fluctuation dominated region.

Then, we investigate how a very small amount of copolymer alters the bending rigidity and the saddle-splay modulus and compare our predictions to the behavior of Gaussian chains at a structureless impenetrable interface [21-23]. If the length of the copolymers exceeds the homopolymer chain length by more than two orders of magnitude, the predictions of the structureless model become fairly accurate. For shorter copolymers there is a pronounced deviation due to the interplay between the copolymer and the other constituents of the interface.

We find here both for a single copolymer and for copolymer mixtures that the structureless interface model tends to overestimate the effect of the copolymer on the bending rigidity. If the chain length of the diblock copolymers is comparable to the size of the homopolymers, the adsorption of a small amount of diblock copolymers even decreases the bending rigidity - in qualitative contrast to the prediction for a structureless interface. A similar effect has been observed in computer simulations by Laradji [40], and we attribute it to the substitution of homopolymers by shorter blocks of copolymers in the interface region.

Recent scattering experiments $[41,45]$ determined the increase of the bending rigidity of an oil-surfactant-water interface upon adsorption of PEP-PEO diblock copolymers. The analysis of the scattering intensity in film contrast [41] implies that-after the renormalization of the bending rigidity due to capillary waves has been taken into account- the structureless interface model overestimates the effect of the diblock by about $30 \%$. This overestimation is in qualitative agreement with our SCF calculations. The analysis of the scattering intensity in bulk contrast [45], however, indicates that the structureless interface model underestimates the effect of the diblock by about $30 \%$. More detailed investigations are clearly needed to clarify this point.
Comparing the elastic constants of an interface with two symmetric and two asymmetric copolymers of corresponding block lengths, we highlight the role of changing the composition of the mixed surfactant layer on the bending rigidity. In the symmetric case, the composition depends only weakly on the curvature $C$, while in the asymmetric case the composition varies linear with $C$ as to increase the amount of asymmetric copolymer with the appropriate spontaneous curvature. Even though the segmental profiles of the symmetric and asymmetric case and the saddle-splay modulus are almost identical, the bending rigidity of the asymmetric copolymer mixture is by a factor as large as 3.4 smaller than for the symmetric copolymer mixture. Once again, our result is in qualitative agreement with simple phenomenological predictions, but the latter fail to provide a quantitatively accurate description. The behavior suggests that a mixture of asymmetric copolymers is advantageous for producing an oil-water microemulsion, because it lowers the saddle-splay modulus and thereby lowers the amount of surfactant required to form a microemulsion and simultaneously lowers the bending rigidity, which in turn destabilizes lamellar order. Experiments $[46,47]$ lend support to this finding.

For symmetric triblock copolymers, our calculations suggest that the middle block gives almost no contribution to the spontaneous curvature, which, in turn, depends only on the properties of the outer blocks, i.e., even if the middle block is much larger, the interface bends towards the middle block and away from the outer blocks. Again, this result is in accord with the properties of a single Gaussian chain [43], which is anchored with both ends onto an elastic sheet. We therefore propose that triblock copolymers provide a very efficient way to tune the spontaneous curvature and induce transitions between microphases with different curvatures.

\section{ACKNOWLEDGMENTS}

It is a great pleasure to thank D. Schwahn, R. Strey, V. Abetz, and D. Richter for stimulating discussions. M.M. thanks the DFG for support (MU-1674/1) and the Forschungszentrum Jülich for friendly hospitality and financial support. CPU time was provided by the NIC Jülich and the computer center of the University of Mainz.
[1] D. Broseta and G. H. Fredrickson, J. Chem. Phys. 93, 2927 (1990).

[2] R. Holyst and M. Schick, J. Chem. Phys. 96, 7728 (1992).

[3] P. K. Janert and M. Schick, Macromolecules 30, 137 (1997).

[4] M. Müller and M. Schick, J. Chem. Phys. 105, 8885 (1996).

[5] F. S. Bates, W. W. Maurer, P. M. Lipic, M. A. Hillmyer, K. Almdal, K. Mortensen, G. H. Fredrickson, and T. P. Lodge, Phys. Rev. Lett. 79, 849 (1997).

[6] H. S. Joen, J. H. Lee, N. P. Balsara, and M. C. Newstein, Macromolecules 31, 3340 (1998).

[7] D. Schwahn, K. Mortensen, H. Frielinghaus, and K. Almdal, Phys. Rev. Lett. 82, 5056 (1999).
[8] D. Schwahn, K. Mortensen, H. Frielinghaus, K. Almdal, and L. Kielhorn, J. Chem. Phys. 112, 5454 (2000).

[9] G. H. Fredrickson and F. S. Bates, J. Polym. Sci., Part B: Polym. Phys. 35, 2775 (1997).

[10] B. Jakobs, T. Sottmann, R. Strey, J. Allgaier, L. Willner, and D. Richter, Langmuir 15, 6707 (1999).

[11] H. Endo, J. Allgaier, G. Gompper, B. Jakobs, M. Monkenbusch, D. Richter, T. Sottmann, and R. Strey, Phys. Rev. Lett. 85, 102 (2000).

[12] R. B. Thompson and M. W. Matsen, Phys. Rev. Lett. 85, 670 (2000).

[13] M. Schick, in Liquids at Interfaces, Proceeding of Les 
Houches, Session XLVIII, edited by J. Charvolin, J. F. Joanny, and J. Zinn-Justin (Elsevier, Amsterdam, 1990).

[14] S. A. Safran and T. Tlusty, Ber. Bunsenges. Phys. Chem. 100, 252 (1996).

[15] D. C. Morse, Curr. Opin. Colloid Interface Sci. 2, 365 (1997).

[16] G. Gompper and D. M. Kroll, Phys. Rev. Lett. 81, 2284 (1998).

[17] W. Helfrich, Z. Naturforsch. C 28, 693 (1973); P. B. Canham, J. Theor. Biol. 26, 61 (1970); E. Evans, Biophys. J. 14, 923 (1974)

[18] M. W. Matsen, J. Chem. Phys. 110, 4658 (1999).

[19] R. B. Thompson and M. W. Matsen, J. Chem. Phys. 112, 6863 (2000).

[20] M. Laradji and R. C. Desai, J. Chem. Phys. 108, 4662 (1998).

[21] C. Hiergeist and R. Lipowsky, J. Phys. II 6, 1465 (1996).

[22] E. Eisenriegler, A. Hanke, and S. Dietrich, Phys. Rev. E 54, 1134 (1996).

[23] C. M. Marques and J. Fournier, Europhys. Lett. 35, 361 (1996).

[24] S. Leibler and D. Andelman, J. Phys. (France) 48, 2013 (1987).

[25] I. Szleifer, D. Kramer, A. Ben-Shaul, W. M. Gelbart, and S. A. Safran, J. Chem. Phys. 92, 6800 (1990).

[26] F. C. MacKintosh and S. A. Safran, Phys. Rev. E 47, 1180 (1993).

[27] U. Seifert, Phys. Rev. Lett. 70, 1335 (1993).

[28] If we did not fix the location of the interface explicitly, the system would be unstable in the grand-canonical ensemble, i.e., the equilibrium would be a homogeneous phase.

[29] E. Helfand, J. Chem. Phys. 62, 999 (1975).

[30] Comparing the saturation line $\sigma=0$ with the complete phase diagram of the ternary system in Ref. [3], we observe that the saturation line is almost identical to the unbinding transition or the homopolymer-rich phase boundary of the three-phase region.

[31] A. N. Semenov, Macromolecules 26, 6617 (1993); 27, 2732 (1994).

[32] A. Werner, F. Schmid, M. Müller, and K. Binder, Phys. Rev. E 59, 728 (1999).

[33] Z.-G. Wang and S. A. Safran, J. Chem. Phys. 94, 679 (1991); J. Phys. (France) 51, 185 (1990).

[34] V. L. Ginzburg, Fiz. Tverd. Tela (Leningrad) 2, 2031 (1960) [Sov. Phys. Solid State 1, 1824 (1960)]; P. G. de Gennes, J.
Phys. (France) Lett. 38, L-441 (1977); J. F. Joanny, J. Phys. A 11, L-117 (1978); K. Binder, Phys. Rev. A 29, 341 (1984).

[35] C. Taupin and P. G. de Gennes, J. Phys. Chem. 86, 2294 (1982).

[36] D. C. Morse, Phys. Rev. E 50, R2423 (1994).

[37] L. Golubović, Phys. Rev. E 50, R2419 (1994).

[38] This criterion applies only if the interaction between the lamellae is negligible, i.e., when the SCF calculations predict highly swollen lamellar phases or when a minor attractive interaction between the interfaces is overcome by repulsion due to fluctuations. Mixtures of copolymers might also prove useful in tuning the interaction between interfaces [12], which is not considered here.

[39] E. M. Blokhuis and D. Bedeaux, J. Chem. Phys. 95, 6986 (1991).

[40] M. Laradji and O. G. Mouritsen, J. Chem. Phys. 112, 8621 (2000).

[41] H. Endo, M. Mihailescu, M. Monkenbusch, J. Allgaier, G. Gompper, D. Richter, B. Jakobs, T. Sottmann, R. Strey, and I. Grillo, J. Chem. Phys. 115, 580 (2001).

[42] G. Porte and C. Ligoure, J. Chem. Phys. 102, 4290 (1995).

[43] C. Hiergeist, Ph.D. thesis, Universität Potsdam, 1997 (unpublished).

[44] The segregation of a chain to the interface is controlled by three contributions to the free energy: (i) the loss of translational entropy, (ii) the lowering of the energy by an amount $2 k_{B} T \chi N_{A}$ per chain due to extending the anchoring blocks into the $A$-rich phase, and (iii) the loss of conformational entropy at the interface. The latter contribution imparts a spontaneous curvature onto the interface. To a first approximation, we conceive the chain conformation of the triblock at the interface as that of individual blocks at an impenetrable surface. Each block suffers an entropy loss of the order $k_{B} T \sqrt{N_{\text {block }}}$. If we increase the size of the middle block $N_{B}$ at fixed $N_{A}$ the loss of conformational entropy will outweigh the enthalpic contribution for $\sqrt{N_{B}}>\chi N_{A}$ and the triblock will be repelled from the interface.

[45] G. Gompper, H. Endo, M. Mihailescu, J. Allgaier, M. Monkenbusch, D. Richter, B. Jakobs, T. Sottmann, and R. Strey, Europhys. Lett. 56, 683 (2001).

[46] R. Strey (private communication).

[47] C. Frank, Diploma thesis, Universität zu Köln, 2001 (unpublished). 